\title{
Voltage-Dependent Anion Channel
}

National Cancer Institute

\section{Source}

National Cancer Institute. Voltage-Dependent Anion Channel. NCI Thesaurus. Code C33887.

The Voltage-Dependent Anion Channel (VDAC) is a small, abundant pore-forming eukaryotic protein in the outer mitochondrial membrane thought to form the major pathway for movement of adenine nucleotides through the outer membrane and to be the mitochondrial binding site for hexokinase and glycerol kinase. 